\title{
Mechanistic Study of Synergistic Antimicrobial Effects between Poly (3-hydroxybutyrate) Oligomer and Polyethylene Glycol
}

\author{
Ziheng Zhang ${ }^{1, \dagger}$, Jun Li ${ }^{1, \dagger}$, Linlin Ma ${ }^{1, \dagger}$, Xingxing Yang ${ }^{1}$, Bin Fei ${ }^{1}$, Polly H. M. \\ Leung ${ }^{2}$ and Xiaoming Tao ${ }^{1, *}$ \\ ${ }^{1}$ Research Center for Smart Wearable Technology, Institute of Textiles and Clothing, \\ The Hong Kong Polytechnic University, Hong Kong, China \\ ${ }^{2}$ Department of Health Technology and Informatics, Hong Kong Polytechnic \\ University, Hong Kong, China \\ $\dagger$ The authors contributed equally to this work \\ * Correspondence: xiao-ming.tao@polyu.edu.hk; Tel.: +852 27666470
}

\begin{abstract}
:
We reported previously that poly (3-hydroxybutyrate) (PHB) oligomer is an effective antimicrobial agent against gram-positive bacteria, gram-negative bacteria, fungi and multi-drug resistant bacteria. In this work, it was further found that polyethylene glycol (PEG) can promote the antimicrobial effect of PHB oligomer synergistically. Three hypothetic mechanisms were proposed, that is, generation of new antimicrobial components, degradation of PHB macromolecules and dissolution/dispersion of PHB oligomer by PEG. With a series of systematic experiments and characterizations of HPLC-MS, it was deducted that dissolution/dispersion of PHB oligomer dominated the synergistic antimicrobial effect between PHB oligomer and PEG. This work demonstrates a way for promoting antimicrobial effect of PHB oligomer and other antimicrobial agents through improving hydrophilicity.
\end{abstract}

Keywords: Poly (3-hydroxybutyric acid); oligomer; polyethylene glycol; antimicrobial agent; synergistic antimicrobial effect. 


\section{Introduction}

Pathogens, e.g. bacteria, fungi and virus, can cause serious diseases due to their high reproductivity and adaptability[1]. Antibiotics have been commonly used to prevent the bacteria infection, whereas the overuse of them lead to antibiotic resistance, which has become a worldwide threat to human health. About 700,000 people die each year due to the infections of multi-drug resistant (MDR) bacteria[2, 3]. Inorganic nanoparticles, e.g. nano-silver particles[2], have been verified to be highly effective to MDR bacteria, nevertheless this type of antimicrobial agents have potential threats to human health and environment[4]. Many new antibiotics and antimicrobial peptides have been developed for dealing with continuously-emerging MDR bacteria, but the developing rate of novel antimicrobial agents cannot catch up with the enhancement of antibiotic resistance of MDR bacteria through bacterial surface modification, protease secretion and expression of efflux pumps[5,6]. It has been found that many essential oils (Eos) were effective to kill MDR bacteria and can be applied with antibiotics for enhance the efficacy synergistically[7, 8]. However, the natural components of EOs cannot be controlled accurately, while the low durability and high volatility restrict their applications.

We have reported previously that the synthesized poly (3-hydroxybutyrate) (S-PHB) oligomer, made by open-ring polymerization of beta-butyrolactone, possessed excellent antimicrobial effect against gram-positive bacteria, gram-negative bacteria and fungi with a high reduction rate over $99.99 \%$, as well as multi-drug resistant (MDR) bacteria (methicillin-resistant S. aureus, ATCC 43300) with reduction rate of 99.97\% [9]. The PHB oligomer is degradable, durable, eco-friendly and safe, thus has significant advantages for the applications in healthcare field. Nevertheless, the material cost of synthesizing PHB (i.e. beta-butyrolactone) is relatively high compared with the fermented PHB powder from starch or sugar, in which the PHB oligomer can be extracted. The extracted PHB (E-PHB) oligomer exhibits a reduction rate of over $90 \%$ against gram-positive bacteria, gram-negative bacteria and fungi. 
In this work, we attempted to improve the antimicrobial property of E-PHB, and found surprisingly that purification reduced the antimicrobial effects of E-PHB, not increased it as expected. We noticed that the elimination of polyethylene glycol (PEG) from the PHB powder extract might be the main reason, a synergistic antimicrobial effect between the PHB oligomer and PEG might exist and should be revealed .

We then proposed three hypothetic mechanisms, including the generation of new antimicrobial components, degradation of PHB macromolecules and dissolution/dispersion of PHB oligomer by PEG during the process of purification. The final experimental results show that the hypothesis of dissolution/dispersion of PHB oligomer by PEG dominates the synergistic antimicrobial effect.

\section{Materials and Methods}

\subsection{Materials}

Poly (3-hydroxybutyrate) (PHB) powder was provided by TianAn Biologic Materials Co., Ltd. in Ningbo, Zhejiang, China. beta-butyrolactone (95.0\%, TCI), aluminium isopropoxide $(98.0 \%, \mathrm{TCI})$ and pyridine $(99.0 \%$, Acros) were used for chemically synthesizing PHB oligomer. PEG $\left(\mathrm{M}_{\mathrm{w}}=600\right.$, Acros Organics $)$ is applied for synergistic antimicrobial effect with PHB oligomer. The solvents, including chloroform, ethanol, methanol, dichloromethane (DCM) and n-hexane, were supplied by Anaqua (Hong Kong).

\subsection{Preparation of PHB oligomer by chemical synthesis}

Beta-butyrolactone $(0.86 \mathrm{~g}, 10 \mathrm{mmol})$ was added to a mixed solution of pyridine $(2 \mathrm{~mL})$ and aluminum isopropoxide $(0.2 \mathrm{~g}, 1 \mathrm{mmol})$, then stirred at $65^{\circ} \mathrm{C}$ under nitrogen atmosphere for $48 \mathrm{~h}[10]$. $\mathrm{HCl}$ solution $(2 \mathrm{~m}, 20 \mathrm{~mL}$ ) was injected to quench the reaction, 
prior to an extraction with DCM. PHB oligomer was obtained after removal of the DCM by vacuum evaporation and separation by column chromatography (DCM/n-hexane = $5: 1, \mathrm{v}: \mathrm{v})[9]$.

2.3 Preparation of PHB oligomer by extraction (E-PHB) and compounding with PEG

Mixture of PHB powder (10 g) and chloroform $(200 \mathrm{~mL})$ was refluxed for $12 \mathrm{~h}$, then 1 $\mathrm{L}$ of methanol was added into the viscous solution, followed by filtration for removing the PHB polymer with high molecular weight (collected as PHB bulk). Afterwards, the raw PHB oligomer was obtained after the removal of solvents through rotary evaporation and further purified by column chromatography (DCM/n-hexane of $1: 1$ to methanol/DCM of 1:2). For compounding, the E-PHB was mixed with PEG (1:1) and stirred under $150{ }^{\circ} \mathrm{C}$ for $4 \mathrm{~h}$.

\subsection{Interaction of PHB powder and PEG}

Bio-based PHB powder was mixed with PEG (1: 1) and stirred under $150{ }^{\circ} \mathrm{C}$ for $4 \mathrm{~h}$. Afterwards, ethanol was added to dissolve the mixture and residual PHB (solid) was removed by centrifugation. After removing the solvent, yellow oil was obtained as a mixture for synergistic antimicrobial test.

2.5 Notions of materials, products and experiments

The materials, products and experiments mentioned in this work are summarized in Table 1. 
Table 1 Notions of materials, products and experiments

\begin{tabular}{|c|c|}
\hline PHB & Poly (3-hydroxybutyrate) \\
\hline PHB powder & Raw material produced by fermentation \\
\hline E-PHB & PHB oligomer extracted from PHB powder \\
\hline EP-PHB & $\begin{array}{l}\text { PHB oligomer extracted from PHB powder followed by } \\
\text { further purification }\end{array}$ \\
\hline S-PHB & PHB oligomer synthesized chemically by polymerization \\
\hline PEG & Polyethylene glycol \\
\hline EP-PHB \& PEG & EP-PHB oligomer reacts with PEG $\left(1: 1,150^{\circ} \mathrm{C}, 4 \mathrm{~h}\right)$ \\
\hline $\begin{array}{l}\text { PHB powder \& } \\
\text { PEG }\end{array}$ & PHB powder reacts with PEG (1:1, room temperature, $4 \mathrm{~h})$ \\
\hline PEG heat treatment & Heat PEG at $150{ }^{\circ} \mathrm{C}$ for $4 \mathrm{~h}$ \\
\hline PHB bulk & $\begin{array}{l}\text { PHB polymer extracted from PHB powder, mainly containing } \\
\text { macromolecules. }\end{array}$ \\
\hline PHB bulk \& PEG & PHB bulk reacts with PEG at $150^{\circ} \mathrm{C}$ for $4 \mathrm{~h}$ \\
\hline $\begin{array}{l}\text { PHB powder heat } \\
\text { treatment }\end{array}$ & Heat PHB powder at $150^{\circ} \mathrm{C}$ for $4 \mathrm{~h}$ \\
\hline $\begin{array}{l}\text { Extract of PHB } \\
\text { powder (methanol) }\end{array}$ & $\begin{array}{l}\text { Extract of PHB powder by methanol dissolution, filtration and } \\
\text { evaporation. }\end{array}$ \\
\hline
\end{tabular}

\subsection{Characterization of PHB and PEG}

HPLC-MS tests were carried out by Thermo Fisher Orbitrap Fusion Lumos Mass Spectrometer. Solvent of methanol/dichloromethane (1: 1, v: v) was adopted as the eluent.

\subsection{Antimicrobial Activity Tests}

The antimicrobial property of the oligomer against Staphylococcus aureus (S. aureus) ATCC No. 6538, Klebsiella pneumoniae (K. pneumoniae) ATCC No. 4352, and 
Candida albicans (C. albicans) ATCC No. 10231 was tested according to the shake flask method[11] with concentration of $10 \mathrm{mg} / \mathrm{mL}[9]$.

\section{Results and discussion}

\subsection{Discovery of synergy between PHB oligomer and PEG}

For producing antimicrobial PHB oligomer with raw materials of lower cost, the extraction of PHB oligomer from PHB powder is preferable compared with chemical synthesis of PHB oligomer. However, the extracted PHB (E-PHB) oligomer possess relatively lower antimicrobial reduction against K. pneumoniae and C. albicans than the synthesized PHB (S-PHB) oligomer, i.e. 94.26\% and 91.95\%, respectively (Table 1). As the PHB oligomer is proved to be an effective antimicrobial agent previously, it is supposed that E-PHB oligomer with higher purity might achieve better antimicrobial effect, thus the E-PHB oligomer is further purified by column chromatography to obtain extracted and purified PHB (EP-PHB) oligomer. Nevertheless, the EP-PHB oligomer with higher purity doesn't possess better antimicrobial effect as expected. Contrarily, it achieves only antibacterial reduction of $49.6 \%$ against $\mathrm{K}$. pneumoniae and has no effect against C. albicans.

For investigating the reason why higher purity leads to low antimicrobial effect, HPLCMS tests are tested for both E-PHB oligomer and EP-PHB oligomer (Figure 3). It can be seen that a polymer component with repeating unit of 44 exists in E-PHB rather than EP-PHB, which is deduced as polyethylene glycol (PEG), as it has been found previously that there are only $\mathrm{C}, \mathrm{H}$ and $\mathrm{O}$ elements exist in the sample[9]. Also, this component appears early $(2 \sim 3.5 \mathrm{~min})$ in the HPLC spectra, which means it has relatively high polarity and further confirm the deduction.

Therefore, it is hypothesized that the exist of PEG in PHB oligomer can promote the antimicrobial effect of PHB, whereas the elimination of PEG after purifying E-PHB decreases its antimicrobial performance. A verifying experiment was carried out to mix EP-PHB oligomer and PEG under $150{ }^{\circ} \mathrm{C}$ for 4 hours and test the antimicrobial effect 
of the prepared mixture, showing a large promoted antimicrobial reduction against $\mathrm{K}$. pneumoniae and C. albicans, i.e. $82.60 \%$ and $85.60 \%$, respectively. The antibacterial reduction of mixture of EP-PHB and PEG against K. pneumoniae $(82.60 \%)$ is higher than that of individual EP-PHB (49.60\%) and PEG (70.00\%). Besides, the raw PHB powder has antibacterial reduction of only $60.28 \%, 0$ and $82.40 \%$ against $\mathrm{S}$. aureus, $\mathrm{K}$. pneumoniae and C. albicans, whereas the mixture of PHB powder and PEG after stirring $4 \mathrm{~h}$ under $150{ }^{\circ} \mathrm{C}$ achieve obviously promoted antibacterial effect, i.e. $92.22 \%$, $99.44 \%$ and $87.70 \%$ for S. aureus, K. pneumoniae and C. albicans, respectively (Table 2 and Figure 2). We further test the antimicrobial effect of S-PHB $(5 \mathrm{mg} / \mathrm{mL})$ and SPHB/PEG (10 mg/mL, $5 \mathrm{mg} / \mathrm{mL}$ for each). As seen in Table 2, compared with $10 \mathrm{mg} / \mathrm{mL}$ S-PHB, the antimicrobial effect of the lower concentration of S-PHB $(5 \mathrm{mg} / \mathrm{mL})$ was significantly reduced, especially C. albicans, whereas S-PHB $(5 \mathrm{mg} / \mathrm{mL})$ achieve much better sterilization effect after mixed with PEG $(5 \mathrm{mg} / \mathrm{ml})$. Therefore, these phenomena confirmed a synergistic antimicrobial effect between PHB oligomer and PEG. 
Table 2 Synergy between PHB oligomer and PEG

\begin{tabular}{|c|c|c|c|c|c|}
\hline \multirow[b]{2}{*}{ Sample } & \multirow{2}{*}{$\begin{array}{l}\text { Concentration } \\
\mathrm{mg} / \mathrm{ml}\end{array}$} & \multirow[b]{2}{*}{$\begin{array}{c}\text { Degree of } \\
\text { polymerization }\end{array}$} & \multicolumn{3}{|c|}{ Antimicrobial property } \\
\hline & & & $\begin{array}{c}\text { S. } \\
\text { aureus }\end{array}$ & $\begin{array}{c}\mathrm{K} . \\
\text { pneumoniae }\end{array}$ & $\begin{array}{c}\text { C. } \\
\text { albicans }\end{array}$ \\
\hline $\begin{array}{c}\text { S-PHB } \\
\text { oligomer } \\
{[9]}\end{array}$ & 10 & $\leqslant 6$ & $>99.99 \%$ & $>99.99 \%$ & $>99.99 \%$ \\
\hline $\begin{array}{c}\text { S-PHB } \\
\text { oligomer }\end{array}$ & 5 & $\leqslant 6$ & $>99.99 \%$ & $91.60 \%$ & $61.50 \%$ \\
\hline $\begin{array}{c}\text { S-PHB } \\
\text { oligomer } \\
\text { and PEG }\end{array}$ & $\begin{array}{c}10 \text { (5 for } \\
\text { each) }\end{array}$ & N.A. & $>99.99 \%$ & $98.30 \%$ & $96.15 \%$ \\
\hline $\begin{array}{c}\text { E-PHB } \\
\text { oligomer } \\
{[9]}\end{array}$ & 10 & $8 \sim 15$ & $>99.99 \%$ & $94.26 \%$ & $91.95 \%$ \\
\hline $\begin{array}{l}\text { EP-PHB } \\
\text { oligomer }\end{array}$ & 10 & $8 \sim 15$ & $>99.99 \%$ & $49.60 \%$ & 0 \\
\hline $\begin{array}{c}\text { EP-PHB } \\
\text { oligomer } \\
\text { and PEG } \\
(1: 1,150 \\
\left.{ }^{\circ} \mathrm{C}, 4 \mathrm{~h}\right)\end{array}$ & $\begin{array}{c}10 \text { (5 for } \\
\text { each) }\end{array}$ & N.A. & $>99.99 \%$ & $82.60 \%$ & $85.60 \%$ \\
\hline PEG & 10 & $\approx 14$ & 0 & $70.00 \%$ & 0 \\
\hline $\begin{array}{c}\text { PHB } \\
\text { powder }\end{array}$ & 10 & $10000 \sim 20000$ & $60.28 \%$ & 0 & $82.40 \%$ \\
\hline $\begin{array}{c}\text { PHB } \\
\text { powder and } \\
\text { PEG }(1: 1, \\
\text { room } \\
\text { temperature } \\
, 4 \mathrm{~h})\end{array}$ & $\begin{array}{c}10 \text { (5 for } \\
\text { each) }\end{array}$ & N.A. & $67.22 \%$ & $79.02 \%$ & $38.50 \%$ \\
\hline $\begin{array}{c}\text { PHB } \\
\text { powder and } \\
\text { PEG } \\
(1: 1,150 \\
\left.{ }^{\circ} \mathrm{C}, 4 \mathrm{~h}\right)\end{array}$ & $\begin{array}{c}10 \text { (5 for } \\
\text { each) }\end{array}$ & N.A. & $92.22 \%$ & $99.44 \%$ & $87.70 \%$ \\
\hline
\end{tabular}



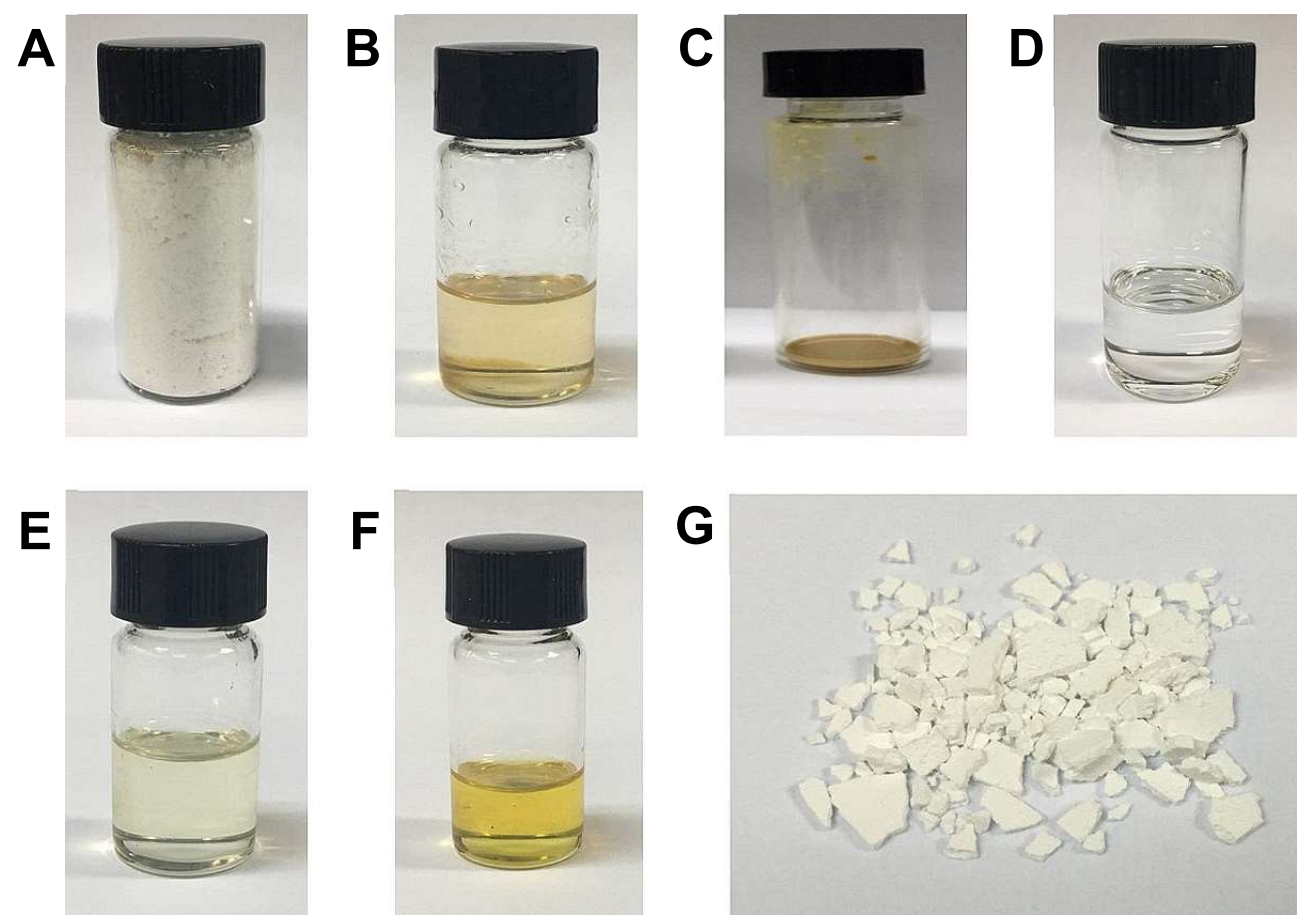

Figure 1 Images of PHB and PEG. (A) PHB powder. (B) S-PHB. (C) E-PHB or EPPHB. (D) PEG. (E) Liquid product of reaction of PHB powder and PEG under room temperature. (F) Liquid product of reaction of PHB powder and PEG under $150{ }^{\circ} \mathrm{C}$. (G) PHB bulk 


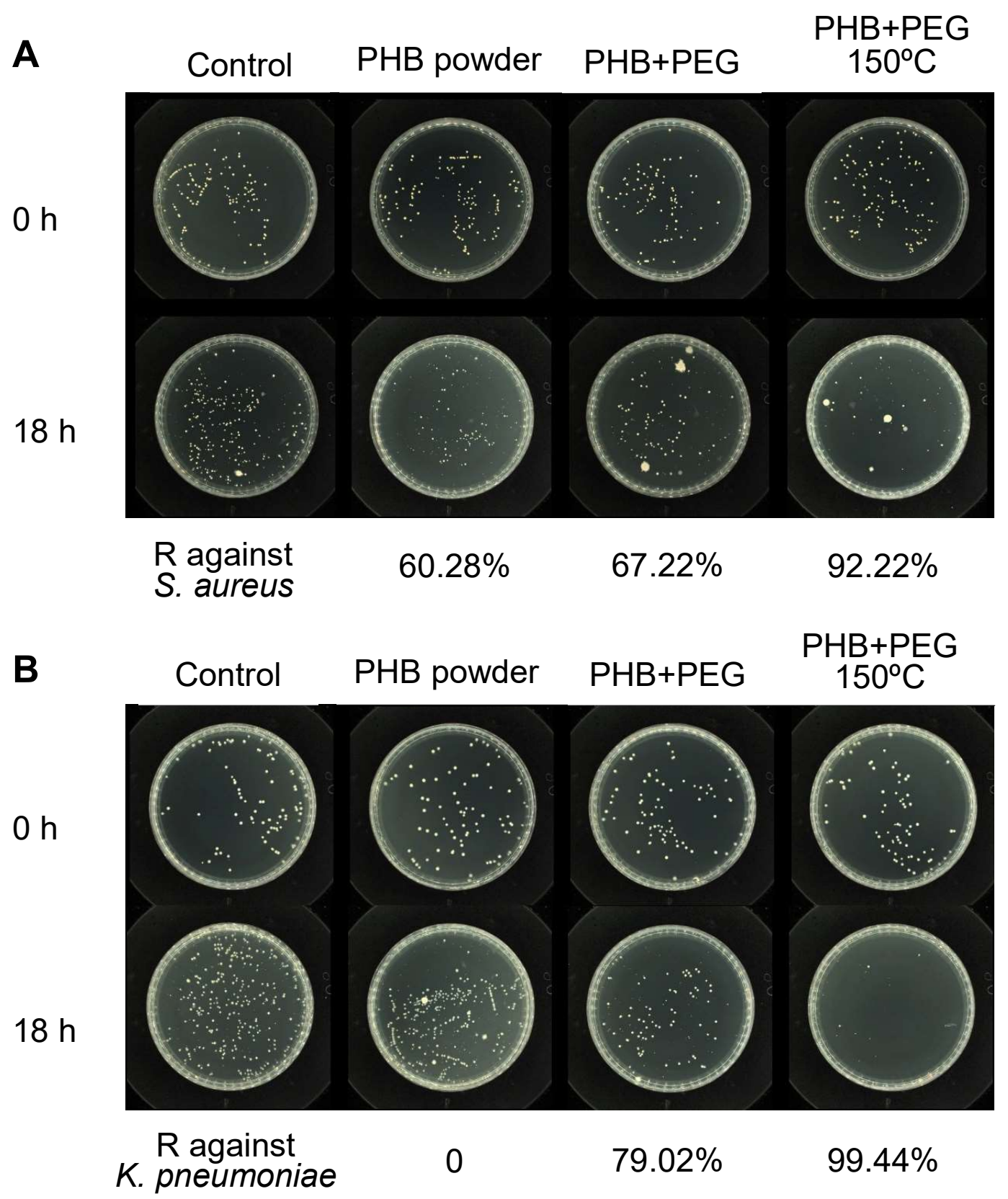

Figure 2 Antimicrobial test for PHB powder, mixture of PHB powder and PEG reacted under room temperature, and mixture of PHB powder and PEG reacted under $150{ }^{\circ} \mathrm{C}$. (A) Antimicrobial test against S. aureus. (B) Antimicrobial test against K. pneumoniae. 


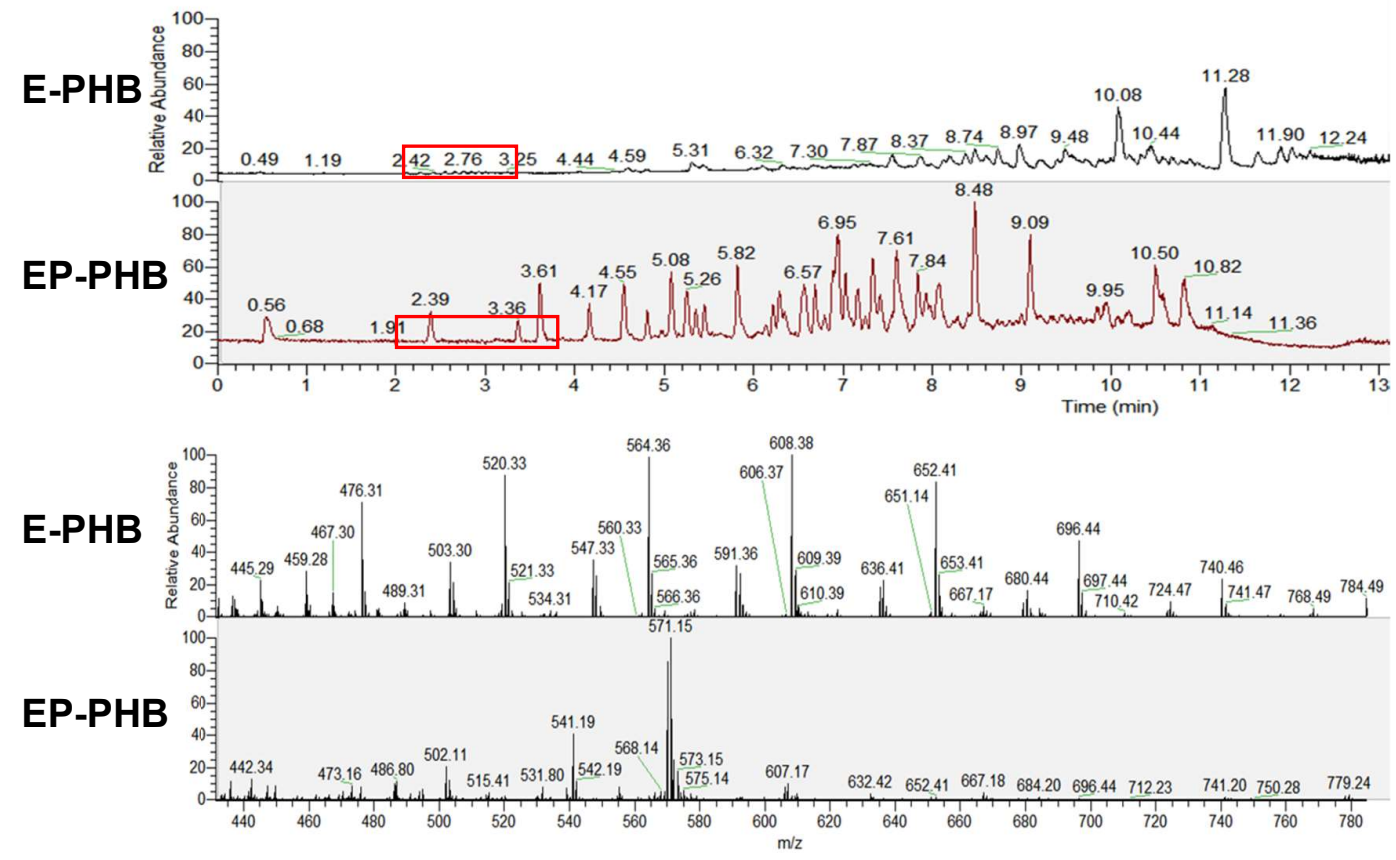

Figure 3 HPLC-MS spectrum of E-PHB and EP-PHB oligomer

3.2 Investigation on synergistic mechanism between PHB oligomer and PEG

After the discovery of synergy between PHB and PEG, three presumptions are raised as generation of new antimicrobial components, degradation of PHB macromolecules and dissolution/dispersion of PHB oligomer by PEG.

\subsubsection{Hypothesis of generation of new antimicrobial components.}

As the mixture of PHB powder and PEG after treatment under $150{ }^{\circ} \mathrm{C}$ possess higher antibacterial reduction than each of the individual component, there might be new components are produced after the heat treatment, which possess better antimicrobial effect than both PHB oligomer and PEG. For verifying this hypothesis, the mass spectrum (MS) is carried out for the solid and liquid part of mixture of PHB powder and PEG after heat treatment, as well as the individual PHB powder and PEG after heat treatment under $150{ }^{\circ} \mathrm{C}$ for $4 \mathrm{~h}$. It can be seen from Figure $4 \mathrm{~A}$ that there is no difference between PHB powder and the solid part of mixture of PHB powder and PEG after heat 
treatment, which means that no new solid antimicrobial component is produced after the reaction of PHB powder and PEG under high temperature. Similarly, the mass spectra of liquid part of mixture of PHB powder and PEG is compared with that of PEG in Figure 4B, showing no new chemical component is produced, whereas the average degree of polymerization of PEG increases approximately from 11 to 14 after heat treatment, illustrating the further polymerization of PEG rather than reaction with PHB power. Therefore, the hypothesis of generation of new antimicrobial components fails due to the constant chemical component observed by MS.
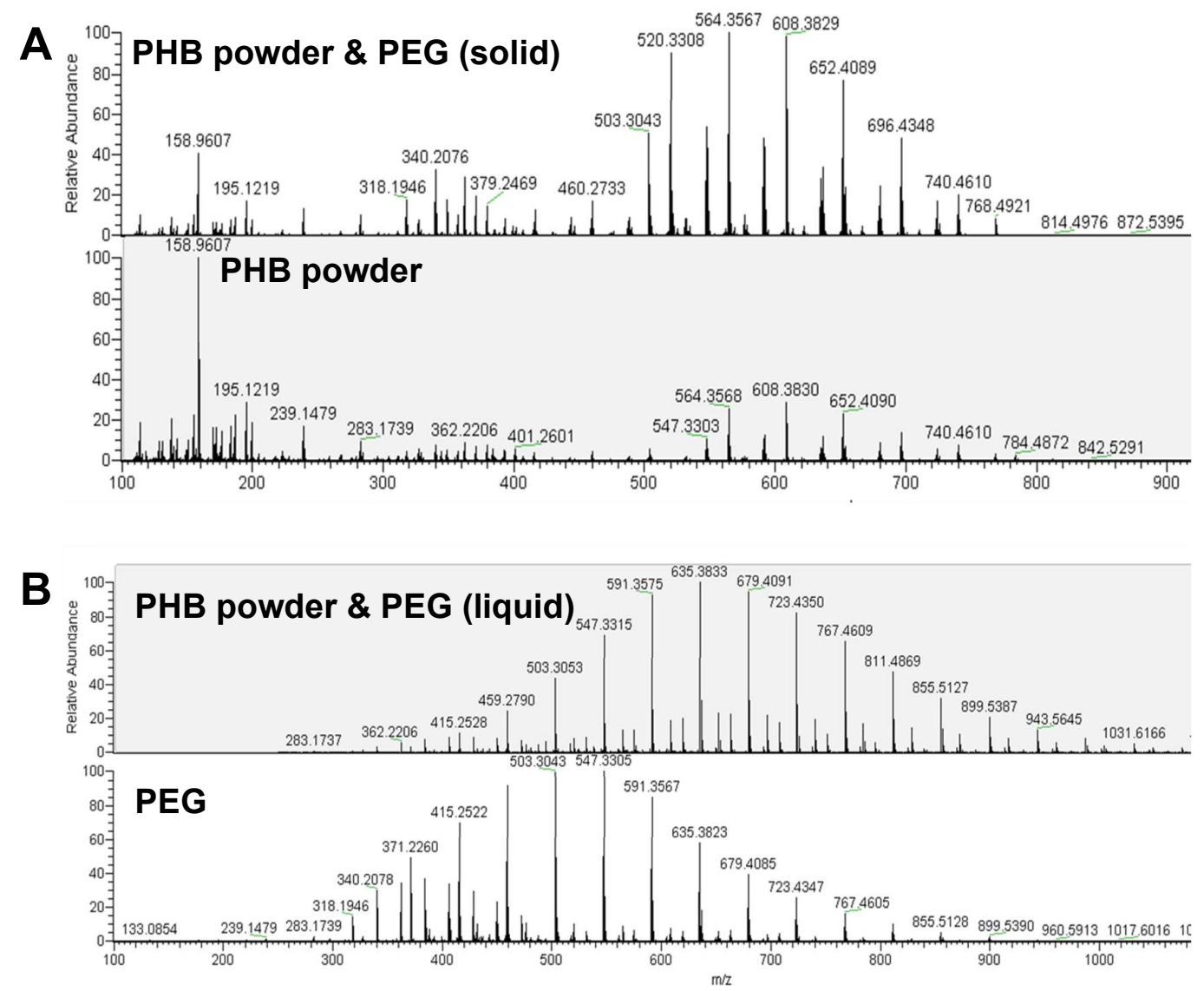

Figure 4 Mass spectrum of PHB powder and PEG after heat treatment. (A) Comparison between PHB power and the solid part of mixture of PHB powder and PEG after heat treatment. (B) Comparison between PEG and the liquid part of mixture of PHB powder and PEG after heat treatment. 


\subsubsection{Hypothesis of degradation of PHB macromolecules}

The second hypothesis is the degradation of PHB macromolecules, which can produce more PHB oligomer for better antimicrobial performance. Actually, it has been found previously that lower degree of polymerization facilitates better antimicrobial effect[9]. Therefore, PHB with higher degree of polymerization, i.e. PHB bulk, was extracted from the raw PHB powder, followed by reaction with PEG under $150{ }^{\circ} \mathrm{C}$ for $4 \mathrm{~h}$, to see if the PHB macromolecules can be degraded by PEG under high temperature. The results are present in the mass spectrum in Figure 5A. It is supposed that PHB oligomer with low degree of polymerization should exist in the liquid part of mixture of PHB bulk and PEG after treatment, whereas there is no peaks of degraded PHB in the spectra, showing almost the same peaks as the spectra of PEG, thus the hypothesis of degradation of PHB macromolecules is also not verified.

Actually, as the melting temperature of $\mathrm{PHB}$ is about $180^{\circ} \mathrm{C}[12]$ and the thermal degradation temperature is over $190{ }^{\circ} \mathrm{C}[13]$, the heat treatment under $150{ }^{\circ} \mathrm{C}$ is not sufficient for degrading the PHB macromolecules, even if in the powder state with high specific surficial area. Figure 5B exhibits the mass spectrum of liquid extract of raw PHB powder before and after heat treatment, showing that only PEG with low degree of polymerization exists, rather than the PHB oligomer through thermal degradation. 

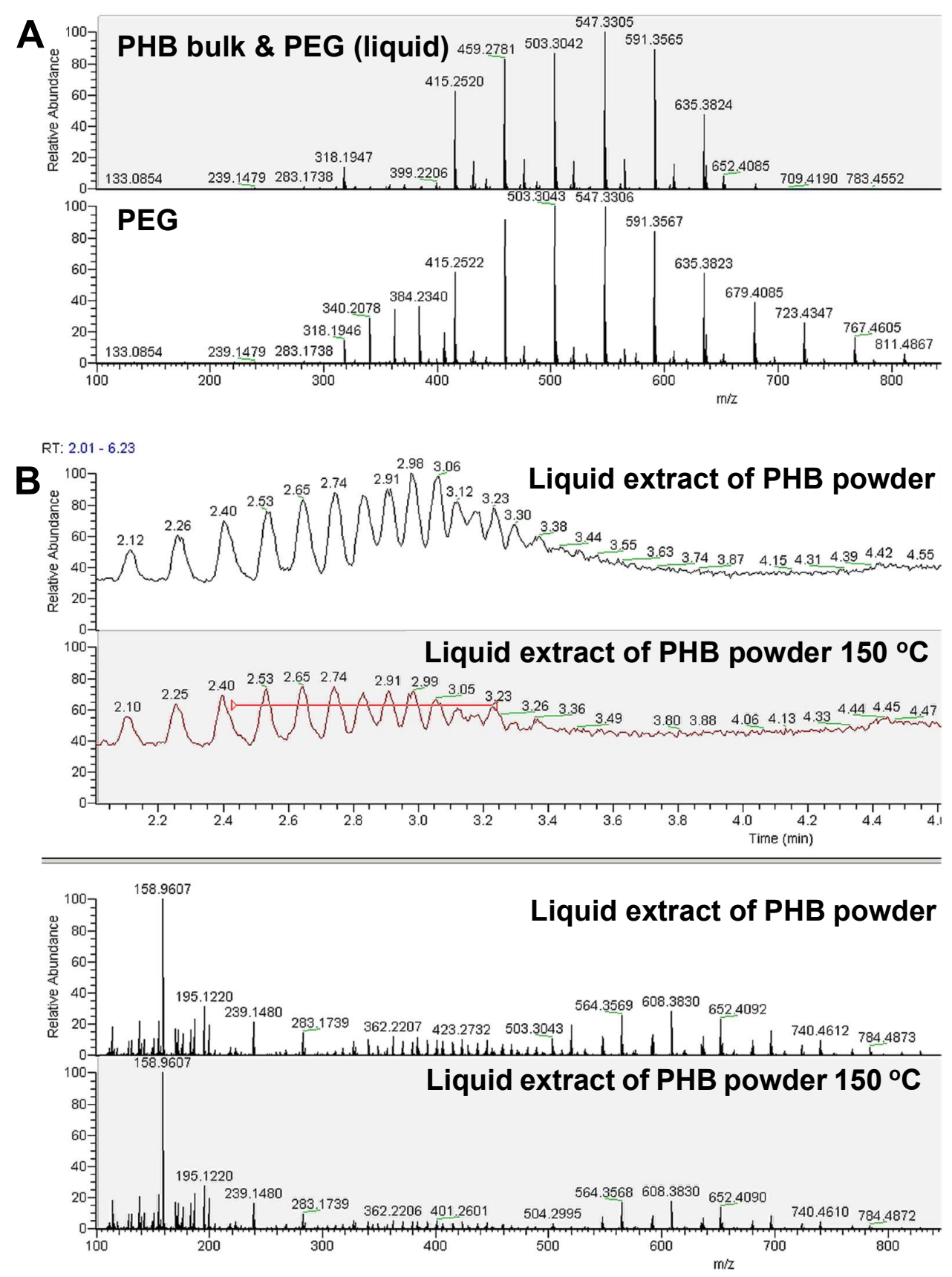

Figure 5 HPLC-MS analysis on hypothesis of degradation of PHB macromolecules. (A) Comparison between PEG and liquid part of mixture of PHB bulk and PEG after treatment under $150{ }^{\circ} \mathrm{C}$ for $4 \mathrm{~h}$. (B) Extract of PHB powder before and after treatment under $150{ }^{\circ} \mathrm{C}$ for $4 \mathrm{~h}$ with solvent of methanol: dichloromethane $=1: 1$.

3.2.3 Hypothesis of dissolution/dispersion of PHB oligomer by PEG 
PEG has very good hydrophilicity and is commonly used for enhancing hydrophilicity of functional materials through compounding $[14,15]$ or grafting[16]. PHB oligomer is relatively hydrophobic material, especially for those with high degree of polymerization. The exist of PEG in raw PHB powder might facilitate the dissolution and dispersion of PHB oligomer therein into water, i.e. antimicrobial solution with bacteria.

For verifying this hypothesis, the PHB bulk (extracted from raw PHB powder with mainly macromolecules) was mixed with PEG (1: 1) prior to a heat treatment under 150 ${ }^{\circ} \mathrm{C}$ for $4 \mathrm{~h}$. The solid part after centrifugation was tested by HPLC-MS with solvent of methanol: dichloromethane $=1$ : 1 (Figure 6A). Compared with the PHB bulk (same condition of treatment and test as above), new regular peaks appear during the eluting time between $6 \mathrm{~min}$ and $10 \mathrm{~min}$, which are determined as PHB oligomer. This phenomenon illustrates that the PEG can promote the dissolution/dispersion of PHB oligomer in bulk into solvent/solution, thus confirm the hypothesis (Figure 7). Though the E-PHB oligomer can be dissolved in methanol, PHB oligomer cannot directly be extracted from raw PHB powder by methanol (only tiny PEG exists in the extract, Figure 6B). Therefore, it is concluded that larger dose of PEG facilitates better affinity of PHB oligomer to solvent by dissolution/dispersion. 

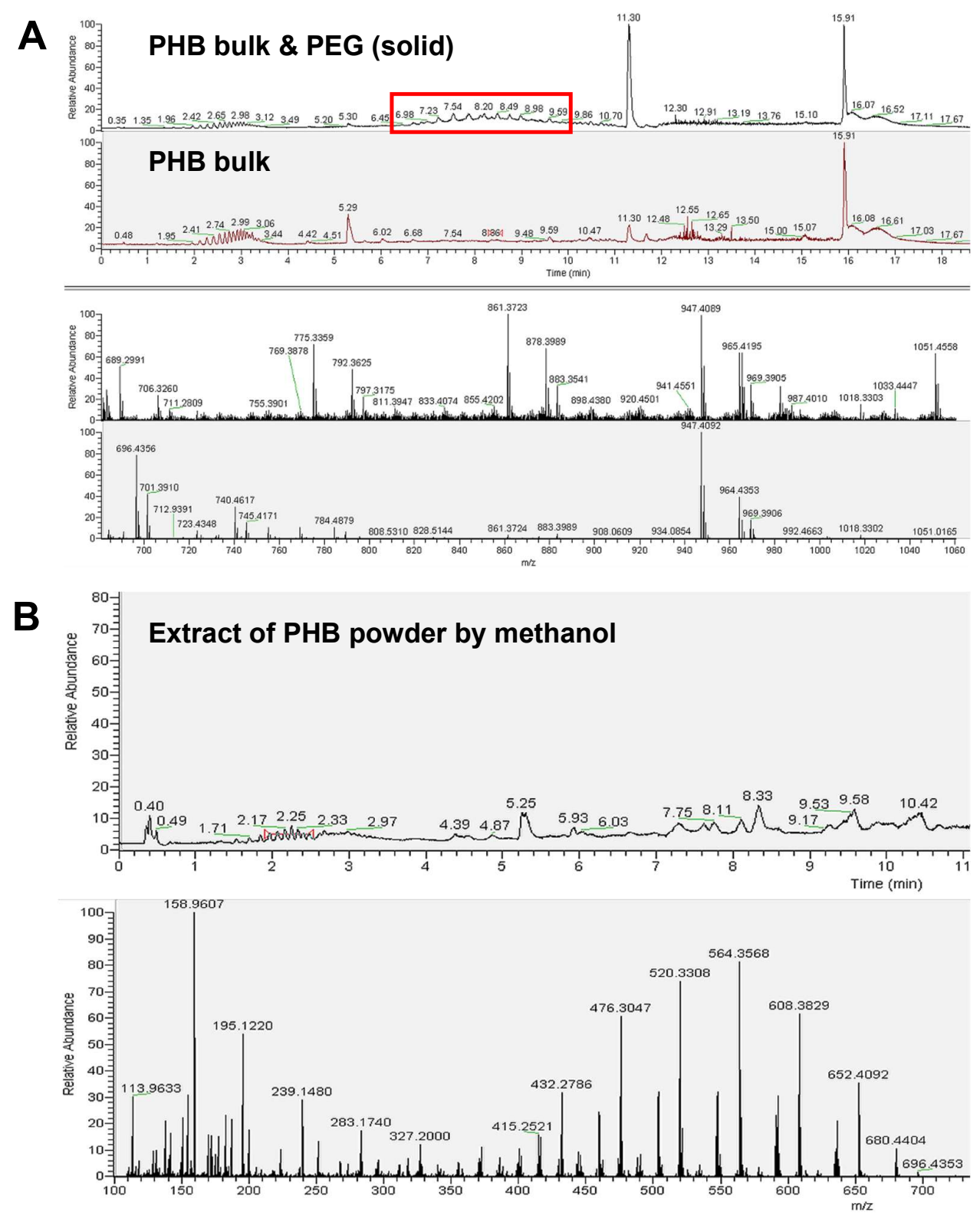

Figure 6 HPLC-MS analysis on hypothesis of dissolution/dispersion of PHB oligomer by PEG. (A) Comparison of PHB bulk and the solid part of mixture of PHB bulk and PEG after heat treatment under $150{ }^{\circ} \mathrm{C}$ for $4 \mathrm{~h}$. (B) Extract of PHB powder with methanol. 


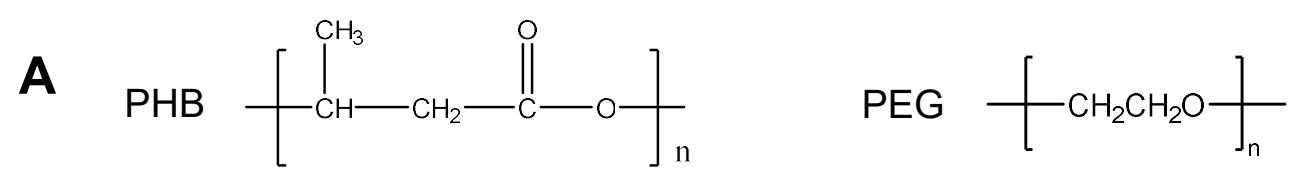

B

PHB bulk $\sim$ PHB macromolecule $\sim$ PHB oligomer $\odot$ PEG

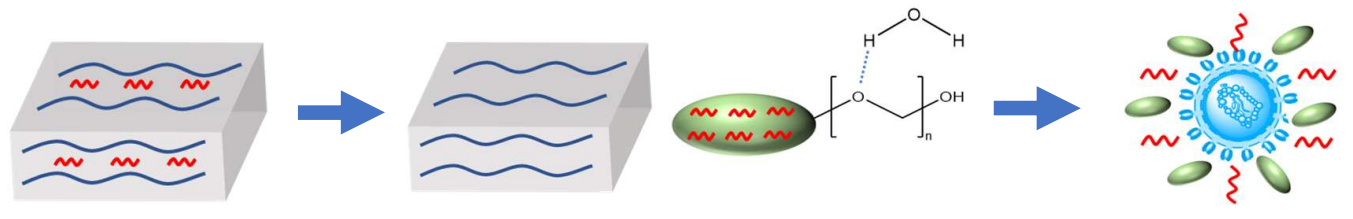

Figure 7 Schematic illustration of hypothesis of dissolution/dispersion of PHB oligomer by PEG. (A) Chemical structure of PHB and PEG. (B) Two steps are presented as dissolution/dispersion and synergistic antimicrobial effect.

\section{Conclusions}

A synergistic antimicrobial effect has been discovered between PHB oligomer and PEG after comparison of a series of antimicrobial tests against gram-positive bacteria, gramnegative bacteria and fungi, followed by HPLC-MS analysis. Three proposed hypothetic mechanisms have been tested by the experiments. The results show that there is neither new produced chemical component nor degradation/condensation of PHB after the compounding of PHB and PEG. The synergistic antimicrobial effect is due to the dissolution/dispersion of PHB oligomer by PEG, which has a good affinity to both the PHB and aqueous antimicrobial solution. This work reveals a new way to produce effective antimicrobial agent from bio-based PHB powder, reduce the minimal inhibitory concentration of pure PHB, and reduce the cost of production. It facilitates the cost-effective applications of bio-based PHB powder and synthesized PHB oligomer through improving the hydrophilicity.

Author Contributions: X.T. initiated, planned, and supervised the execution of the research. Z.Z. and J.L. implemented the extraction, chemical reaction and 
characterization. L.M. and X.Y. conducted the antimicrobial tests. B.F. supervised the chemical analysis and synthesis work. P.H.M.L. supervised the microbiological study.

Funding: This work was supported by the Innovation and Technology Commission of SAR Government of Hong Kong (Grant No. ITP/039/16TP).

Conflicts of Interest: The authors declare no conflict of interest.

\section{References}

1. Feng, Y.; Liu, L.; Zhang, J.; Aslan, H.; Dong, M. Photoactive antimicrobial nanomaterials. J Mater Chem B 2017, 5, 8631-8652.

2. Zheng, K.; Setyawati, M. I.; Leong, D. T.; Xie, J. Antimicrobial silver nanomaterials. Coord. Chem. Rev. 2018, 357, 1-17.

3. Willyard, C. The drug-resistant bacteria that pose the greatest health threats. Nature News 2017, 543, 15.

4. Gao, Y.; Yang, T.; Jin, J. Nanoparticle pollution and associated increasing potential risks on environment and human health: a case study of China. Environ. Sci. Pollut. Res. Int. 2015, 22, 19297-306.

5. Chaudhary, A. S. A review of global initiatives to fight antibiotic resistance and recent antibiotics' discovery. Acta Pharmaceutica Sinica B 2016, 6, 552-556.

6. Guilhelmelli, F.; Vilela, N.; Albuquerque, P.; Derengowski, L.; Silva-Pereira, I.; Kyaw, C. Antibiotic development challenges: the various mechanisms of action of antimicrobial peptides and of bacterial resistance. Frontiers in microbiology 2013, 4, 353.

7. Simoes, M.; Bennett, R. N.; Rosa, E. A. Understanding antimicrobial activities of phytochemicals against multidrug resistant bacteria and biofilms. Nat. Prod. Rep. 2009, 26, 746-757.

8. Langeveld, W. T.; Veldhuizen, E. J.; Burt, S. A. Synergy between essential oil components and antibiotics: a review. Crit Rev Microbiol 2014, 40, 76-94. 
9. Ma, L.; Zhang, Z.; Li, J.; Yang, X.; Fei, B.; Leung, P. H. M.; Tao, X. M. A new antimicrobial agent: poly (3-hydroxybutyric acid) oligomer. Macromol. Biosci. 2019, 19, e1800432.

10. Matsubara, K.; Eda, K.; Ikutake, Y.; Dan, M.; Tanizaki, N.; Koga, Y.; Yasuniwa, M. Aluminum complex initiated copolymerization of lactones and DL - lactide to form crystalline gradient block copolymers containing stereoblock lactyl chains. J. Polym. Sci., Part A: Polym. Chem. 2016, 54, 2536-2544.

11. Hauenstein, O.; Agarwal, S.; Greiner, A. Bio-based polycarbonate as synthetic toolbox. Nature communications 2016, 7, 1-7.

12. Abdelwahab, M. A.; Flynn, A.; Chiou, B.-S.; Imam, S.; Orts, W.; Chiellini, E. Thermal, mechanical and morphological characterization of plasticized PLAPHB blends. Polym. Degradation Stab. 2012, 97, 1822-1828.

13. Janigova, I.; Lacı'k, I.; k, I. C. Thermal degradation of plasticized poly(3hydroxybutyrate) investigated by DSC. Polym. Degradation Stab. 2002, 77, 35 41.

14. Rekik, S. B.; Gassara, S.; Bouaziz, J.; Deratani, A.; Baklouti, S. Enhancing hydrophilicity and permeation flux of chitosan/kaolin composite membranes by using polyethylene glycol as porogen. Appl Clay Sci 2019, 168, 312-323.

15. Kiani, S.; Mousavi, S. M.; Shahtahmassebi, N.; Saljoughi, E. Hydrophilicity improvement in polyphenylsulfone nanofibrous filtration membranes through addition of polyethylene glycol. ApSS 2015, 359, 252-258.

16. He, M.; Zhang, R.; Zhang, K.; Liu, Y.; Su, Y.; Jiang, Z. Reduced graphene oxide aerogel membranes fabricated through hydrogen bond mediation for highly efficient oil/water separation. J Mater Chem A 2019, 7, 11468-11477. 